\title{
Removing a mixture of Gaussian and impulsive noise using the total variation functional and split Bregman iterative method
}

\author{
Bishnu P. Lamichhane ${ }^{1}$
}

(Received 19 February 2015; revised 11 October 2015)

\begin{abstract}
We apply the split Bregman iterative method to minimise the total variation of a piecewise polynomial function to remove Gaussian and impulsive noise from an image. We compare these numerical results with another approach based on the gradient penalty. Both approaches use a finite element method. Numerical results show that the method based on the total variation functional is superior only for one class of images.
\end{abstract}

http://journal.austms.org.au/ojs/index.php/ANZIAMJ/article/view/9316 gives this article, (C) Austral. Mathematical Soc. 2015. Published October 27, 2015, as part of the Proceedings of the 17th Biennial Computational Techniques and Applications Conference. ISSN 1446-8735. (Print two pages per sheet of paper.) Copies of this article must not be made otherwise available on the internet; instead link directly to this URL for this article. 


\section{Contents}

1 Introduction

C53

2 The mixture of Gaussian and impulsive noise

C54

3 Total variation smoothing using a finite element method

C56

4 Numerical results

C59

5 Conclusion

C62

References

C62

\section{Introduction}

One of the very important problems in image processing is how to recover a clear image out of a noisy image. Although it is very difficult to know the model governing the noise, the most common noise types encountered in real applications are impulsive noise, Gaussian noise or a mixture of both. More details concerning digital image processing are found elsewhere [1, 2, 3]. In this article, we consider a mixture of impulsive and Gaussian noise. We apply a finite element method to remove the noise using a total variation (TV) minimisation functional and split Bregman iterative solution method. Finite element methods have recently become popular in different areas of image processing $[4,5,6,7,8]$.

While there are many efficient approaches to remove impulsive noise or Gaussian noise from an image [9, 6, 10, 11, 12, 13], removing a mixture of Gaussian and impulsive noise is more difficult. To recover a clear image when the original image is corrupted with a mixture noise, we use a finite element smoothing based on the minimisation of a functional involving the total variation of a piecewise polynomial function. Section 3 introduces the 
finite element smoothing technique based on the minimisation of a functional involving the total variation and an approach for solving the minimisation problem is given.

A digital image is an array of numbers, where the size of the array determines the size of the image. An intensity image, for example, is a single matrix of size $m \times n$, where each element of the matrix represents the intensity of the image pixel or grey level. These intensity images are also called black and white images. On the other hand, an array of size $m \times n \times 3$ describes a colour image where the image has the size $m \times n$, and each pixel has three values representing the red, green and blue intensities that make up the colour. In this article, we restrict ourselves to intensity images.

We consider salt and pepper noise as an example of impulsive noise. The advantage of this noise is that the pixel location of an impulse can be identified. Salt and pepper noise is caused by transmission errors, where the corrupted pixels are randomly set either to zero or the maximum value, leading to a 'salt and pepper' like appearance in the image. Here the zero value denotes black and the maximum value denotes white in an intensity image [2]. Our approach is applicable for a general impulsive noise after using an impulse detector $[9,6,10]$ to identify the impulsive pixels.

\section{The mixture of Gaussian and impulsive noise}

If a noisy image is transmitted over faulty communication lines, then the received image might be corrupted with a mixture of Gaussian and impulsive noise. For an image corrupted with a mixture of Gaussian and impulsive noise, it is necessary to smooth the image pixels as well as remove impulses. For example, Garnett et al. [10] used a noise filter based on a local image statistic to remove a mixture of noise, whereas Xu et al. [14] used a fuzzy based method, and Lamichane [15] used a finite element method with a gradient 
penalty.

Let $\Omega=[0,1] \times[0,1]$. We explain the mixture of noise using a set of points. Define a set of points $\mathcal{S} \subset \Omega$ in an image where

$$
\mathcal{S}=\left\{\left(a_{i}, b_{j}\right)\right\}_{i=1, j=1}^{n, m} \quad \text { with } \quad a_{i}=\frac{i-1}{n-1} \quad \text { and } \quad b_{j}=\frac{j-1}{m-1} .
$$

We also define an image function $\mathrm{I}_{\mathrm{f}}: \mathcal{S} \rightarrow \mathbb{R}^{k}, k \in \mathbb{N}$, where, for a colour image $k=3$ and for an intensity image $k=1$. In a colour image $I_{f}$ gives the three values, red, green and blue, at $\left(a_{i}, b_{j}\right)$ and in an intensity image $I_{f}$ gives the intensity at $\left(a_{i}, b_{j}\right)$. For intensity images, the number $I_{f}\left(a_{i}, b_{j}\right)$ is either defined to be in $[0,1]$ or in $[0,255]$. In either case, the smallest intensity represents black, and the largest intensity represents white.

Let $\tilde{I}$ be the image corrupted with a mixture of Gaussian and impulsive noise, and let $\mathrm{I}$ be the original image. We associate the set of points $\mathcal{S}$ as defined in (1) with both images, and image functions $\mathrm{I}_{f}: \mathcal{S} \rightarrow \mathbb{R}$ and $\tilde{\mathrm{I}}_{\mathrm{f}}: \mathcal{S} \rightarrow \mathbb{R}$ with the images I and $\tilde{\mathrm{I}}$, respectively. Let $\mathcal{S}^{n}$ and $\mathcal{S}^{p}$ be the set of points corrupted with impulsive noise and non-corrupted, respectively, with $\mathcal{S}=\mathcal{S}^{n} \cup \mathcal{S}^{p}$. As the positions of the noisy pixels are random, the points in $\mathcal{S}^{p}$ have no structure.

Since the image is corrupted with a mixture of Gaussian and impulsive noise, after removing the impulsive pixels from the noisy image

$$
\tilde{\mathrm{I}}_{\mathrm{f}}\left(\mathrm{a}_{i}, \mathrm{~b}_{\mathrm{j}}\right)=\mathrm{I}_{\mathrm{f}}\left(\mathrm{a}_{\mathrm{i}}, \mathrm{b}_{j}\right)+\mathrm{n}_{\mathrm{ij}}, \quad\left(\mathrm{a}_{i}, \mathrm{~b}_{j}\right) \in \mathcal{S}^{\mathfrak{p}},
$$

where the $\mathfrak{n}_{\mathrm{ij}}$ are drawn from a zero-mean Gaussian distribution. Hence our problem is to fit a smooth surface to the scattered data in $\mathcal{S}^{p}$. In the following, we denote the set of points in $\mathcal{S}^{p}$ by $\left\{\left(x_{i}, y_{i}\right)\right\}_{i=1}^{N}$ and $\left\{z_{i}=\tilde{I}_{f}\left(x_{i}, y_{i}\right)\right\}_{i=1}^{N}$. 


\section{Total variation smoothing using a finite element method}

Radial basis functions and thin plate splines are often used to interpolate and smooth scattered data $[16,17]$. Here, we consider an approach based on minimising the total variation of the image function, which requires minimising the functional

$$
\frac{\lambda}{2} \sum_{i=1}^{N}\left[u\left(x_{i}, y_{i}\right)-z_{i}\right]^{2}+\int_{\Omega}|\nabla u| d x d y,
$$

over a suitable space of functions, where $u$ is an interpolant, $N$ is the number of uncorrupted pixels in the image, $\nabla$ is the gradient operator, and $\lambda$ is a positive constant called a smoothing parameter. The gradient penalty approach is obtained by replacing $\int_{\Omega}|\nabla \mathfrak{u}| \mathrm{d} x \mathrm{dy}$ with $\int_{\Omega}|\nabla u|^{2} \mathrm{~d} x \mathrm{~d} \boldsymbol{y}$ in (2), leading to a linear approach [15].

The motivation of our approach is that, although the given data is completely unstructured because of the random positions of the impulses, we want to reconstruct the image in a structured grid.

Let $C^{0}(\Omega)$ be the space of continuous functions in $\Omega$, where $\Omega$ is the unit square. Let $\mathcal{T}$ be a structured decomposition of the unit square $\Omega$ into rectangles or triangles using the set of points $\mathcal{S}$, where rectangles and triangles are obtained by joining points in $\mathcal{S}$. Note that $\mathcal{T}$ is the set of triangles and rectangles. Then let

$$
\mathrm{V}=\left\{\mathrm{u} \in \mathrm{C}^{0}(\Omega):\left.u\right|_{\mathrm{T}} \in \mathrm{P}(\mathrm{T}), \mathrm{T} \in \mathcal{T}\right\}
$$

be a finite element space, where $|u|_{T}$ is the restriction of $u$ on $T, P(T)$ is the linear polynomial space if $\mathrm{T}$ is a triangle and the bilinear polynomial space on $T$ if $T$ is a rectangle [18]. Now our discrete problem is to minimise the functional (2) over the function space $\mathbf{V}$ so that the discrete problem is

$$
\min _{\mathfrak{u} \in V} \frac{\lambda}{2} \sum_{i=1}^{N}\left[u\left(x_{i}, y_{i}\right)-z_{i}\right]^{2}+\int_{\Omega}|\nabla u| d x d y .
$$


We aim to solve this problem using the split Bregman method proposed by Goldstein and Osher [19], which is one of the most efficient approaches to solve the total variation minimisation problem. To this end we approximate the total variation integral over an element $T \in \mathcal{T}$ as

$$
\int_{\mathrm{T}}|\nabla u| d x d y \approx|T| \sqrt{\left(\partial_{x} u\right)_{T}^{2}+\left(\partial_{y} u\right)_{T}^{2}},
$$

where $\left(\partial_{x} \mathfrak{u}\right)_{T}$ and $\left(\partial_{y} u\right)_{T}$ are partial derivatives of $\boldsymbol{u}$ with respect to $\boldsymbol{x}$ and $\boldsymbol{y}$ evaluated at the centroid of T, respectively. Since

$$
\int_{\Omega}|\nabla u| d x d y=\sum_{T \in \mathcal{T}} \int_{T}|\nabla u| d x d y
$$

we have the approximation

$$
\int_{\Omega}|\nabla u| d x d y \approx \sum_{T \in \mathcal{T}}|T| \sqrt{\left(\partial_{x} u\right)_{T}^{2}+\left(\partial_{y} u\right)_{T}^{2}} .
$$

Let $\mathrm{Q}$ be the space of piecewise functions with respect to the mesh $\mathcal{T}$. We now introduce two piecewise constant functions $D_{x}$ and $D_{y}$ in $Q$ with respect to the mesh $\mathcal{T}$, defined as

$$
\left.D_{x}\right|_{T}=\sqrt{|T|}\left(\partial_{x} u\right)_{T} \quad \text { and }\left.\quad D_{y}\right|_{T}=\sqrt{|T|}\left(\partial_{y} u\right)_{T} \quad \text { for } \quad T \in \mathcal{T} .
$$

The vector of all $u$ at the corrupted points is

$$
P u=\left(u\left(x_{0}, y_{0}\right), u\left(x_{1}, y_{1}\right), \ldots, u\left(x_{N}, y_{N}\right)\right),
$$

and the vector $\mathbf{z}=\left(z_{1}, z_{2}, \ldots, z_{\mathrm{N}}\right)$. We want to solve

$$
\underset{\mathbf{u} \in \mathrm{V}}{\arg \min }\left[\frac{\lambda}{2}\|\mathrm{Pu}-\mathbf{z}\|^{2}+\|\mathrm{D}(\mathrm{u})\|_{1}\right],
$$

where $\|\cdot\|$ is the Euclidean norm and $D(u)=\left(D_{x}, D_{y}\right)$ with

$$
\|\mathrm{D}(\mathrm{u})\|_{1}=\left\|\left(\mathrm{D}_{x}, \mathrm{D}_{y}\right)\right\|_{1}=\sum_{\mathrm{T} \in \mathcal{T}}|\mathrm{T}| \sqrt{\left(\partial_{x} u\right)_{T}^{2}+\left(\nabla_{y} u\right)_{\mathrm{T}}^{2}} .
$$


The idea of the split Bregman iteration is to split the minimisation problem (5) into two minimisation problems which are solved separately [19]. This is done by introducing two additional unknown functions $d_{x}$ and $d_{y}$ to approximate $D_{x}$ and $D_{y}$, and then performing the following steps, increasing $k$ after each loop until convergence, after initialising $d^{k}, u^{k}$ and $b^{k}$ for $k=0$. Here $d^{k}, b^{k} \in Q$, whereas $u^{k}$ is the vector representation of a finite element function in $V$ for $k \in \mathbb{N}_{0}$.

1. For tolerance $\epsilon$, while $\left\|u^{k}-u^{k+1}\right\|>\epsilon$ repeat the following steps;

2. $u^{k+1}=\arg \min _{u \in V} \lambda\left\|P u^{k}-z\right\|^{2}+\mu\left\|d^{k}-D\left(u^{k}\right)-b^{k}\right\|_{2}^{2}$;

3. $\mathrm{d}^{\mathrm{k}+1}=\arg \min _{\mathrm{d} \in \mathrm{Q}}\|\mathrm{d}\|_{1}+\mu\left\|\mathrm{d}-\mathrm{D}\left(\mathrm{u}^{\mathrm{k}+1}\right)-\mathrm{b}^{\mathrm{k}}\right\|_{2}^{2}$;

4. $b^{k+1}=b^{k}+D\left(u^{k+1}\right)-d^{k+1}$.

The above iteration finds a fixed point $\left(u^{*}, d^{*}, b^{*}\right)$ of

$$
\begin{aligned}
\left(u^{k+1}, d^{k+1}\right) & =\underset{u, d}{\arg \min }\|d\|_{1}+\lambda\|P u-z\|^{2}+\mu\left\|d-D(u)-b^{k}\right\|_{2}^{2}, \\
b^{k+1} & =b^{k}+D\left(u^{k+1}\right)-d^{k+1},
\end{aligned}
$$

so that the fixed point $b^{*}$ satisfies $b^{*}=b^{*}+D\left(u^{*}\right)-d^{*}$ leading to $D\left(u^{*}\right)=$ $\mathrm{d}^{*}[19]$.

Step 2 is a differentiable optimisation problem and is therefore solved by using the conjugate gradient or other iterative methods. It is sufficient to perform just a few conjugate gradient iterations in this step. Step 3 is solved by using shrinkage [19]. One of the big advantages of the split Bregman algorithm over the continuation method [20] is that the parameter $\mu$ in the above algorithm can be safely chosen to be a constant [19]. We have set $\mu=1$ and $\epsilon=10^{-3}$ in the following numerical results. 


\section{Numerical results}

Real-life images Tables 1 and 2 show the peak signal-to-noise ratios for the reconstructed images, where the images are corrupted with both Gaussian and salt and pepper noises. The peak signal-to-noise ratio is defined as

$$
\mathrm{PSNR}=10 \log _{10}\left(\frac{\max _{\mathrm{I}}^{2}}{\mathrm{MSE}}\right)=20 \log _{10}\left(\frac{\max _{\mathrm{I}}}{\sqrt{\mathrm{MSE}}}\right),
$$

where $\max _{\mathrm{I}}$ is the maximum pixel value of the image, and MSE is the mean square error, that is,

$$
\operatorname{MSE}=\frac{1}{m n} \sum_{i=1}^{m} \sum_{j=1}^{n}\left\|I_{f}\left(a_{i}, b_{j}\right)-\hat{\mathrm{I}}_{f}\left(a_{i}, b_{j}\right)\right\|^{2} .
$$

Here, $I_{f}$ is the image function for the original image and $\hat{I}_{f}$ is the image function for the image recovered after removing the noise. We consider Gaussian noise with mean zero and variances $\sigma=0.05$ and $\sigma=0.1$, as shown in Tables 1 and 2, respectively. Salt and pepper noise with densities 50\%, $60 \%$ and $70 \%$ are applied. We compare two different methods of removing a mixture of Gaussian and salt and pepper noises. To find the optimal value of $\lambda$ we run the test code with different $\lambda$ and choose the one yielding the best PSNR. We see that there is not much difference between the two methods: the gradient penalty method and total variation method [21].

Figure 1 shows recovered images for a Baboon image using Gaussian noise of variance 0.05 and impulsive noise of density $60 \%$. We see that the image recovered by using the TV method looks a bit sharper than the one recovered by the gradient penalty. However, the peak signal to noise ratios are very similar for both approaches.

Figure 2 shows recovered images for a peppers image using the same noise as in the baboon image. As in the previous baboon example, the TV method produces a slightly sharper image than the gradient penalty method. 
Table 1: PSNR of the test images with various noise densities (Gaussian and salt and pepper noise, $\sigma=0.05$ ).

\begin{tabular}{l|ccc|ccc} 
& \multicolumn{3}{|c|}{$\begin{array}{c}\text { PSNR for Baboon image } \\
\text { Noise Density }\end{array}$} & \multicolumn{3}{c}{ PSNR for Peppers image } \\
& $50 \%$ & $60 \%$ & $70 \%$ & $50 \%$ & $60 \%$ & $70 \%$ \\
\hline Denoising method & 18.82 & 18.57 & 18.45 & 22.21 & 22.02 & 21.63 \\
Gradient penalty & 18.62 & 18.62 & 18.51 & 21.79 & 21.19 & 19.72
\end{tabular}

Table 2: PSNR of the test images with various noise densities (Gaussian and salt and pepper noise, $\sigma=0.1$ ).

\begin{tabular}{l|ccc|ccc} 
& \multicolumn{3}{|c|}{$\begin{array}{c}\text { PSNR for Baboon image } \\
\text { Noise density }\end{array}$} & \multicolumn{3}{c}{ PSNR for Peppers image } \\
& $50 \%$ & $60 \%$ & $70 \%$ & $50 \%$ & $60 \%$ & $70 \%$ \\
\hline Denoising method & 17.95 & 18.07 & 17.88 & 20.73 & 20.51 & 20.26 \\
Gradient penalty & 18.32 & 18.01 & 17.51 & 21.04 & 20.94 & 19.47
\end{tabular}

Figure 1: Baboon image (first), noisy image (second), image recovered by the TV method (third) and by the gradient penalty method (last).

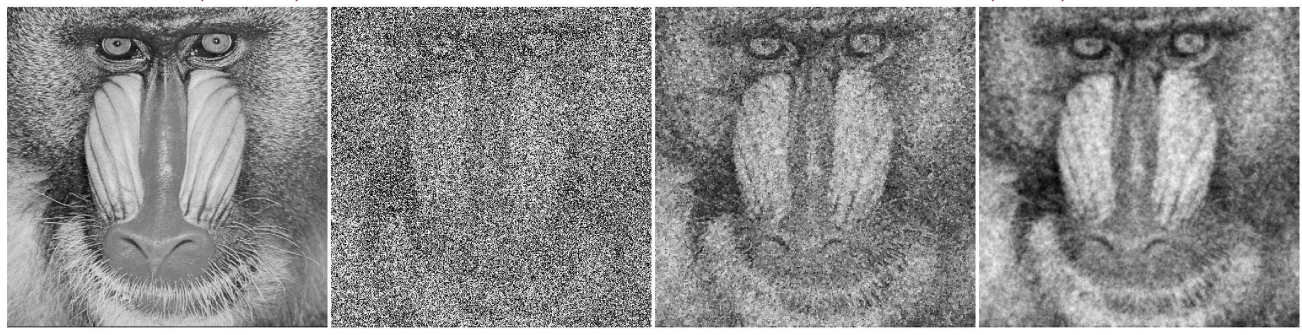


Figure 2: Peppers image (first), noisy image (second), image recovered by the TV method (third) and by the gradient penalty method (last).
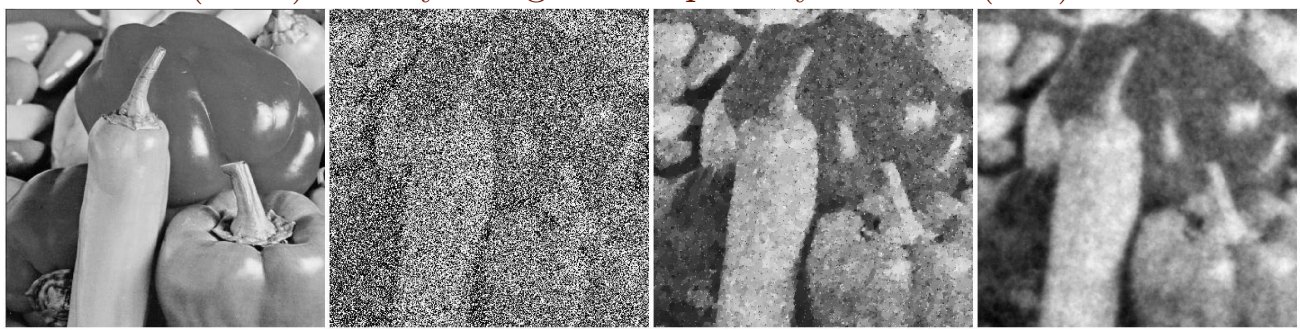

Figure 3: Apple image (first), noisy image (second), image recovered by the TV method (third) and by the gradient penalty method (last).
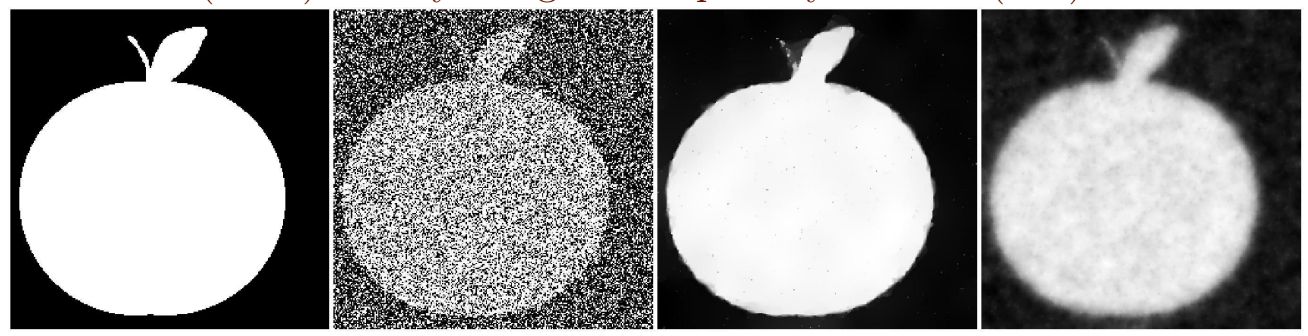

Binary images We now perform numerical analyses of the binary images. Intensity images with only black and white pixels are called binary images. It is well-known that total variation image denoising does an excellent job of preserving the sharpness of an image [2]. Figure 3 shows the numerical results for a binary image using the same noise as in the previous examples. In this example, we see the strong visual difference between the TV method and the gradient penalty method.

Table 3 shows the peak signal to noise ratios for the binary test image using Gaussian noise with mean zero and variances $\sigma=0.05$ and $\sigma=0.1$. We see the superiority of the total variation method as peak signal to noise ratios are significantly better for the total variation method.

Finally, we investigate the convergence of the split Bregman algorithm with 
Table 3: PSNR of the binary test image with various noise densities (Gaussian and salt and pepper noise)

\begin{tabular}{l|ccc|ccc} 
& \multicolumn{3}{|c|}{ PSNR with $\sigma=0.05$} & \multicolumn{3}{c}{ PSNR with $\sigma=0.1$} \\
& \multicolumn{3}{|c|}{ Noise density } & \multicolumn{3}{c}{ Noise density } \\
\hline Denoising method & $50 \%$ & $60 \%$ & $70 \%$ & $50 \%$ & $60 \%$ & $70 \%$ \\
Gradient penalty & 16.61 & 16.21 & 15.91 & 17.88 & 14.73 & 14.39 \\
Total variation & 22.30 & 22.11 & 21.51 & 19.90 & 19.74 & 19.37
\end{tabular}

respect to $\lambda$. Figures 4, 5 and 6 show the number of iterations of the split Bregman algorithm and PSNR for different values of $\lambda$ for all three test images. We see that the number of iterations is small when we have higher PSNR.

\section{Conclusion}

We presented a method of removing a mixture of impulsive and Gaussian noise from images using a total variation functional. The total variation functional is discretised by using a finite element method and solved by the split Bregman iterative method. Numerical results demonstrate that the approach based on the total variation penalty is superior to the gradient penalty approach only for images with sharp jump discontinuities.

\section{References}

[1] Plataniotis, K. N. and Venetsanopoulos, A. N. Color Image Processing and Applications. Springer, 2000. doi:10.1007/978-3-662-04186-4 C53

[2] Chan, T. F. and Shen, J. Image Processing And Analysis: Variational, PDE, Wavelet, And Stochastic Methods. SIAM, 2005. doi:10.1137/1.9780898717877 C53, C54, C61 
Figure 4: Number of iterations and peak signal-to-noise ratio versus $\lambda$ : Baboon image.

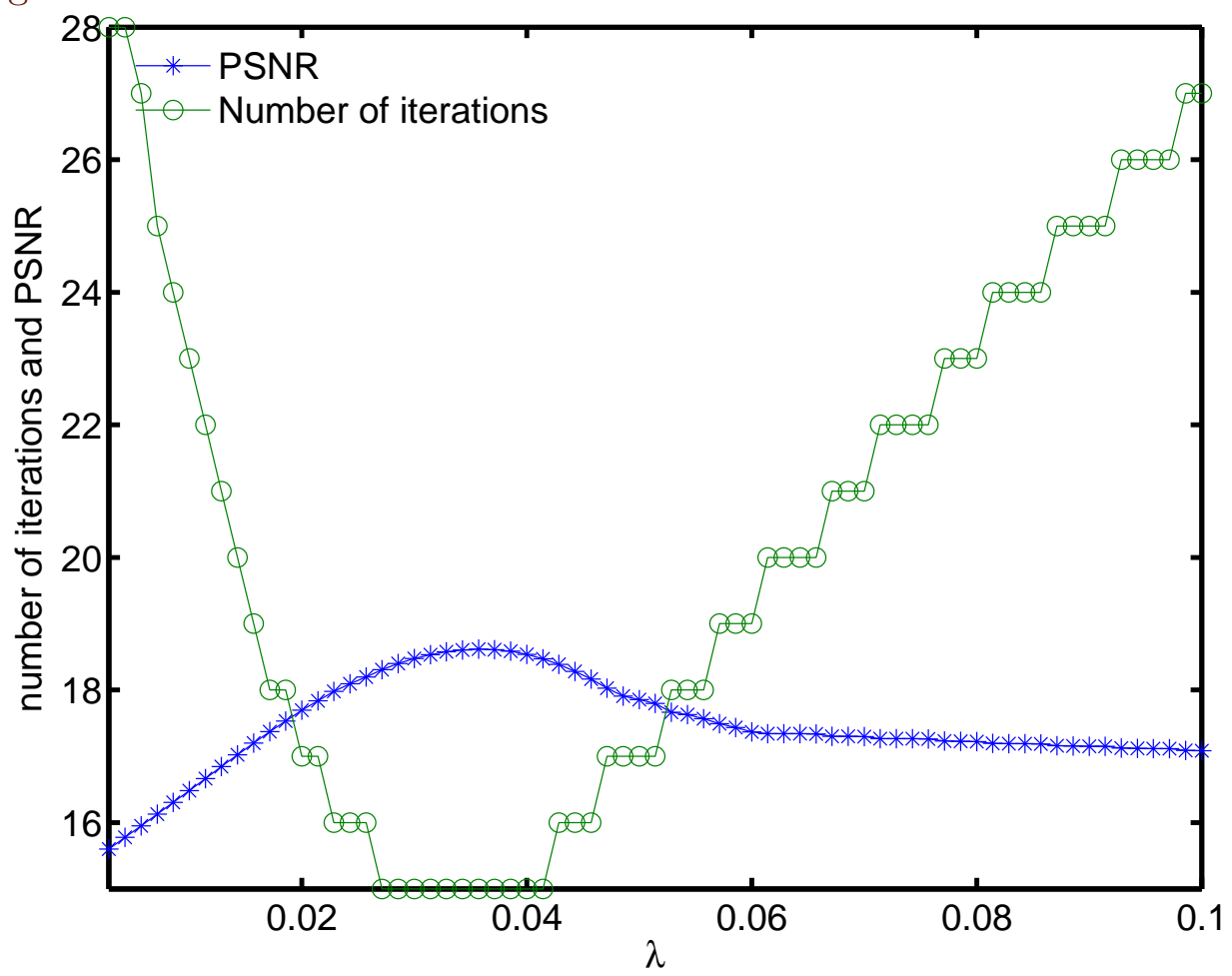

[3] Blanchet, G. and Charbit, M. Digital Signal and Image Processing using Matlab. Wiley, 2010. doi:10.1002/9780470612385. C53

[4] Preusser, T. and Rumpf, M. An adaptive finite element method for large scale image processing. J. Vis. Commun. Image R. 11:183-195 2000. doi:10.1006/jvci.1999.0444 C53

[5] Ferrant, M., Warfield, S. K., Nabavi, A., Jolesz, F. A. and Kikinis, R. Registration of 3D intraoperative MR images of the brain using a finite-element biomechanical model. IEEE T. Med. Imaging 20:1384-1397, 2001. doi:10.1007/978-3-540-40899-4_3 C53 
Figure 5: Number of iterations and peak signal-to-noise ratio versus $\lambda$ : peppers image.

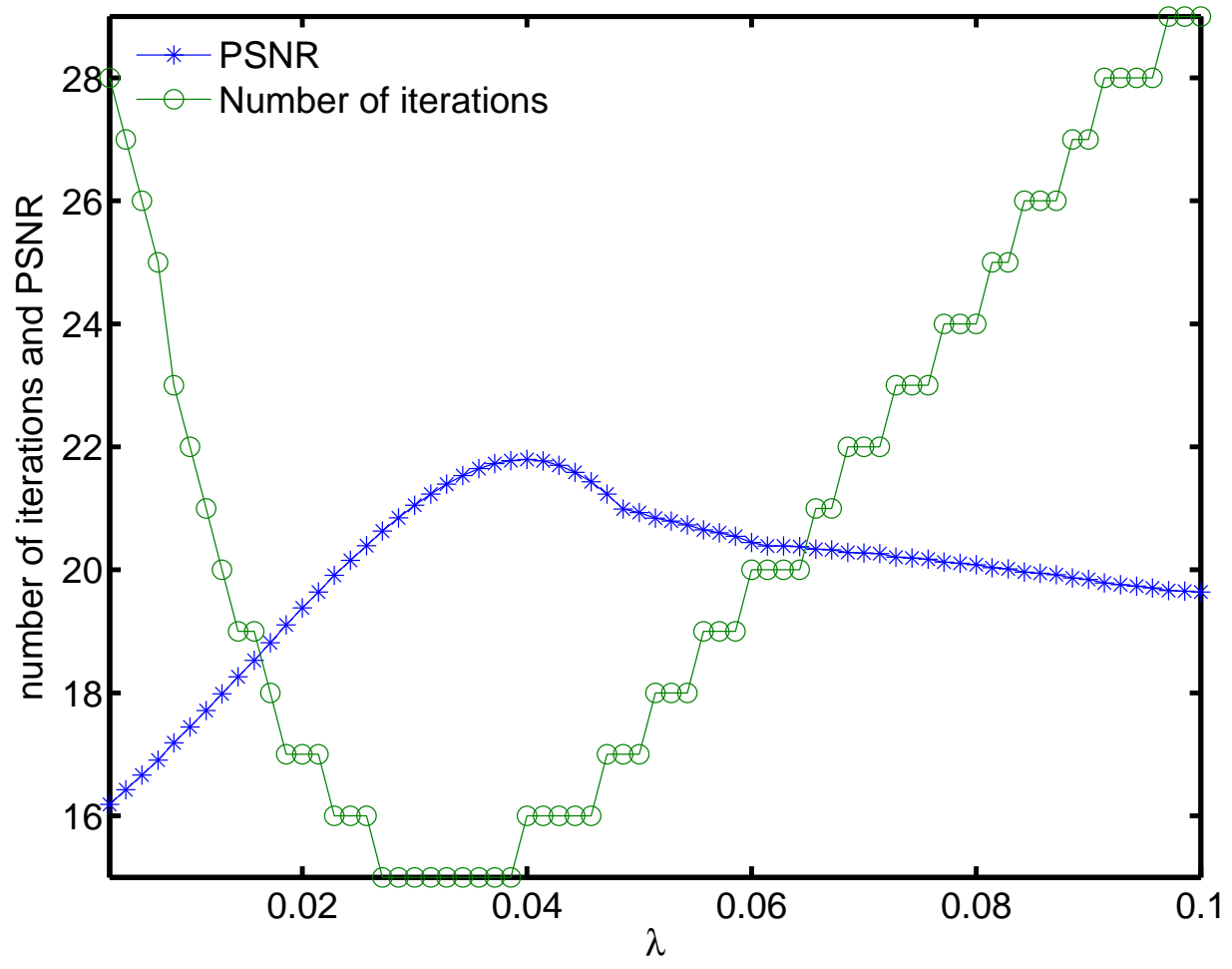

[6] Besdok, E. Impulsive noise suppression from images by using Anfis interpolant and Lillietest. EURASIP J. Appl. Sig. P. 2004:526574, 2004. doi:10.1155/S1110865704403126 C53, C54

[7] Wang, Z., Qi, F. and Zhou, F. A discontinuous finite element method for image denoising. In Image Analysis and Recognition vol. 4141 of Lecture Notes in Computer Science. Springer Berlin/Heidelberg, 2006. doi:10.1007/11867586_11 C53

[8] Demaret, L. and Iske, A. Adaptive image approximation by linear splines over locally optimal Delaunay triangulations. IEEE Signal Proc. Lett. 
Figure 6: Number of iterations and peak signal-to-noise ratio versus $\lambda$ : apple image.

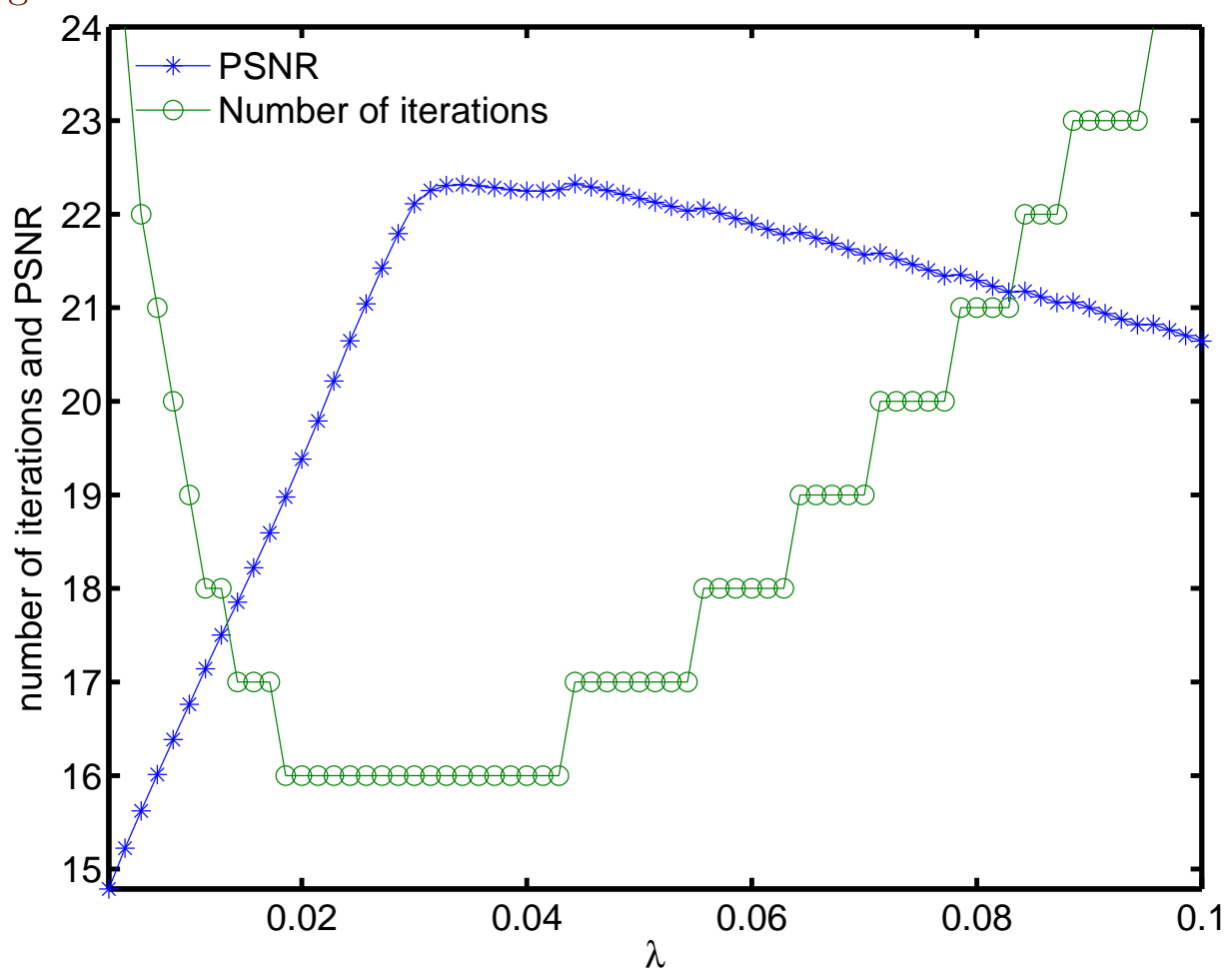

13:281-284, 2006. doi:10.1109/LSP.2006.870358 C53

[9] Aizenberg, I. and Butakoff, C. Effective impulse detector based on rank-order criteria. IEEE Signal Proc. Lett. 11:363-366, 2004. doi:10.1109/LSP.2003.822925. C53, C54

[10] Garnett, R., Huegerich, T., Chui, C. and He, W. A universal noise removal algorithm with an impulse detector. IEEE T. Image Process. 14:1747-1754, 2005. doi:10.1109/TIP.2005.857261 C53, C54

[11] Donoho, D. L. De-noising by soft-thresholding. IEEE T. Inform. 
Theory 41:613-627, 1995. doi:10.1109/18.382009. C53

[12] Donoho, D. L. and Johnstone, J. M. Ideal spatial adaptation by wavelet shrinkage. Biometrika 81:425-455, 1994. doi:10.1093/biomet/81.3.425 C53

[13] Luisier, F., Blu, T. and Unser, M. A new SURE approach to image denoising: interscale orthonormal wavelet thresholding. IEEE T. Image Process. 16:593-606, 2007. doi:10.1109/TIP.2007.891064 C53

[14] Xu, Q., Ma, L., Li, M., Wang, W., Cai, J., Brunelli, R. and Messelodi, S. Fuzzy weighted average filtering for mixture noises. In Third International Conference on Image and Graphics pp. 18-21, 2004. doi:10.1109/ICIG.2004.70 C54

[15] Lamichhane, B. P. Finite element techniques for removing mixture of gaussian and impulsive noise. IEEE T. Signal Process. 57:2538-2547, 2009. doi:10.1109/TSP.2009.2016272 C54, C56

[16] Duchon, J. Splines minimizing rotation-invariant semi-norms in Sobolev spaces. In Constructive Theory of Functions of Several Variables, Lecture Notes in Mathematics 571:85-100. Springer-Verlag Berlin, 1977. doi:10.1007/BFb0086566 C56

[17] Wahba, G. Spline Models for Observational Data, vol. 59, of Series in Applied Mathematic. SIAM, Philadelphia, 1990. doi:10.1137/1.9781611970128 C56

[18] Quarteroni, A. and Valli, A. Numerical approximation of partial differential equations. Springer-Verlag, Berlin, 1994.

doi:10.1007/978-3-540-85268-1 C56

[19] Goldstein, T. and Osher, S. The split Bregman method for L1-regularized problems. SIAM J. Imaging Sci. 2:323-343, 2009. doi:10.1137/080725891 C57, C58 
[20] Wang, Y., Yang, J., Yin, W. and Zhang, Y. A new alternating minimization algorithm for total variation image reconstruction. SIAM J. Imaging Sci. 1:248-272, 2008. doi:10.1137/080724265 C58

[21] van den Doel, K., Ascher, U. M. and Haber, E. The lost honour of 12-based regularization. In Large Scale Inverse Problems Radon Series on Computational and Applied Mathematics 13:181-203. De Gruyter, 2012. http://www.degruyter.com/viewbooktoc/product/182025 C59

\section{Author address}

1. Bishnu P. Lamichhane, School of Mathematical \& Physical Sciences, Mathematics Building - V127, University of Newcastle, University Drive, Callaghan, NSW 2308, Australia mailto:Bishnu.Lamichhane@newcastle.edu . au 